\title{
Continuum Arm Robotic Manipulator: A Review
}

\author{
Puneet Kumar Singh ", C. Murali Krishna \\ Department of Mechanical Engineering, M.A.N.I.T Bhopal \\ *Corresponding Author: puneetsingh_nitb@live.com
}

Copyright (C) 2014 Horizon Research Publishing All rights reserved.

\begin{abstract}
Traditional robots have rigid underlying structures that limit their ability to interact with their environment. For example, conventional robot manipulators have rigid links and can manipulate objects using only their specialised end effectors. These robots often encounter difficulties operating in unstructured and highly congested environments. A variety of animals and plants exhibit complex movement with soft structures devoid of rigid components. In this paper survey of literature related to continuum arm of robotic manipulator based on biological inspiration is carried out. It defines the fundamental difference between discrete, serpentine and continuum robot devices; presents the 'state of the art' of continuum robots and outlines their areas of application; and introduces some control issues. Finally some conclusions regarding the continued development of these devices are made.
\end{abstract}

Keywords Biologically Inspired Robot, Continuum Robot, Trunk, Tentacle, Snake Locomotion

\section{Introduction}

Robotic manipulators have very versatile use in Automobile, Medical, space exploration, Search and Rescue, underwater exploration and in Military. There are many different kinds of robots, factory automation systems that weld and assemble car engines, examine and inspect cracks in parts, machines that's place chocolate into boxes, medical devices that support surgeons in operations requiring high-precision manipulation, cars that drive automatically over long distances, vehicles for planetary exploration, mechanism for power line or oil platform inspection, service robots, In a sense, all these robots are inspired by biological system. Robots are used to help in dangerous, monotonous, and tedious jobs. Most of the existing robotic manipulators are designed and build in a manner to maximize stiffness in an attempt to minimize the vibration of the end-effector to achieve good position accuracy. This high stiffness is achieved by using heavy material and a bulky design. Hence, the existing heavy rigid manipulators are to be inefficient in terms of power consumption or speed with respect to the operating payload. Also, the operation of high precision robots is severely limited by their dynamic deflection, which persists for a period of time after a move is completed.

In order to improve industrial productivity, it is required to reduce the weight of the arms and/or to increase their speed of operation. For these purposes it is very desirable to build flexible robotic manipulators. Compared to the conventional heavy and bulky robots, flexible manipulators have the potential advantage of lower cost, larger work volume, higher operational speed, greater payload-to-manipulator-weight ratio, smaller actuators, lower energy consumption, better maneuverability, better transportability and safer operation due to reduced inertia. But the greatest disadvantage in flexible manipulators arises when one tries to track a specific tip position trajectory by applying the torques at the actuation ends. These dexterous robotic arms are classified into two categories, i.e., discrete and continuum. Discrete robots consist of a series of short rigid links connected to one another with the use of discrete joints. As more links are connected, the degree of freedom for the robot will increase. The high degree of freedom motion enables them to bend in any direction and the motion of these robots is highly predictable. Unfortunately, the increased number of links will increase the overall weight as well. Unlike discrete robots, continuum robots generate smooth curving motions similar to snakes via elastic deformation. Generally, continuum robots have a much lighter body structure than their discrete counterparts but the motion of the continuum robots is more unpredictable.

\section{Continuum (Biologically Inspired) Robotic Manipulator}

Most robot manipulators feature a structure inspired by that of the human arm, in the sense of being based on a serial arrangement of rigid links, connected by a relatively small (six or fewer) degrees of freedom. In this way, traditional robot manipulators are essentially "vertebrate" structures. However, traditional robots - like humans - encounter difficulties operating in highly congested environments, or in manipulating objects with parts of their arm other than their specialized end effector. These problems have been effectively addressed in nature by structures such as elephant trunks, mammal, locomotion of snake, lizard tongues and 
octopus arms. These highly successful muscular structures feature significant compliance and adaptability.

Continuum robots are inspired by the biological world. These robots feature a backbone-less structure similar to such biological counterparts as snakes, elephant trunks and octopus arms. Like these organisms, a continuum robot can use the entire length of its arm to grasp objects of different shapes and sizes. It wraps its body around the object until it has a firm grip on the object, similar to an elephant using its trunk to move logs. Potential applications of continuum robots include navigation through congested and unpredictable environments where a continuum robot can be used for underground or underwater exploration. A continuum robot with its unique abilities can reach places that are usually inaccessible for rigid link robots and hostile for human beings. These robots are also known for their wide range of grasping abilities. They can firmly grasp objects of different shapes and sizes. The grasping can also be performed by various biologically inspired methods.
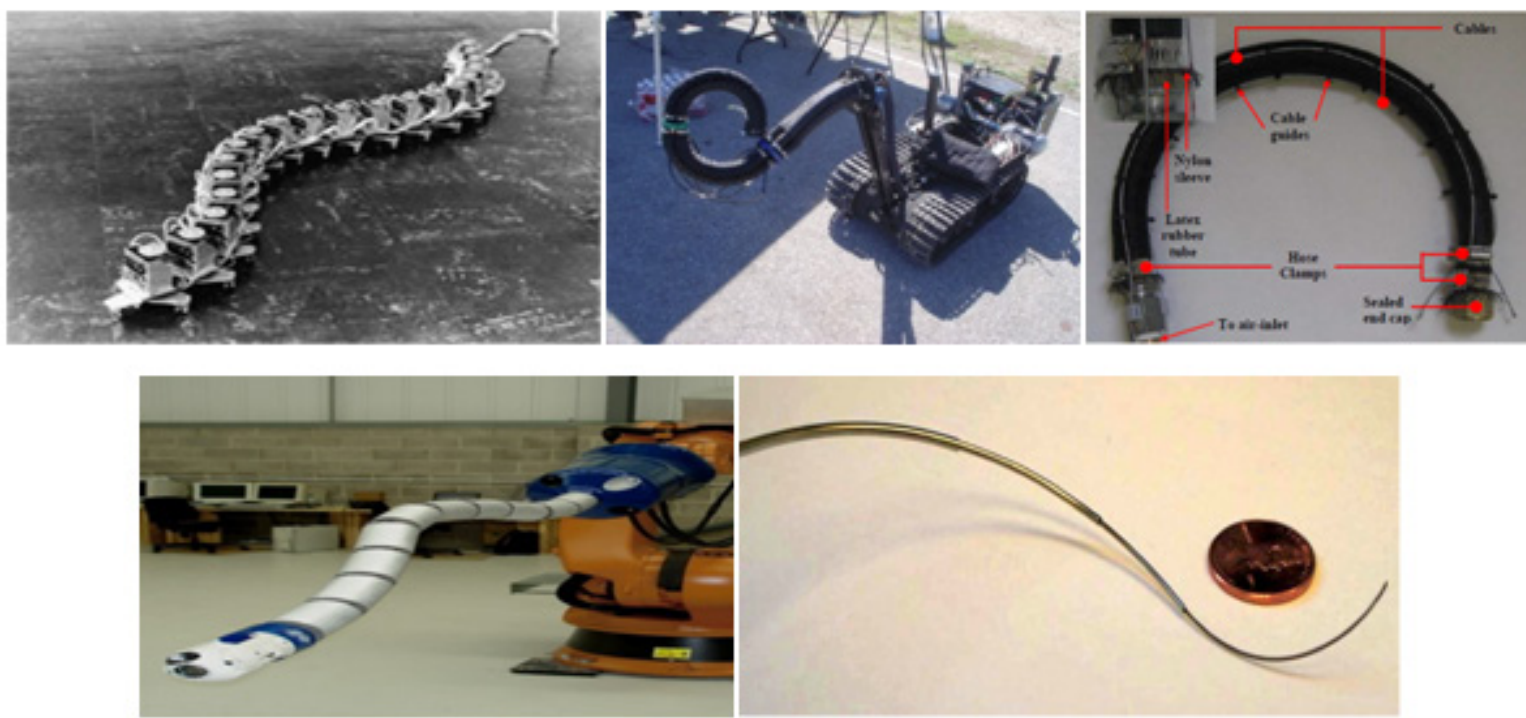

Figure 1. Already developed bio-Inspired Robots

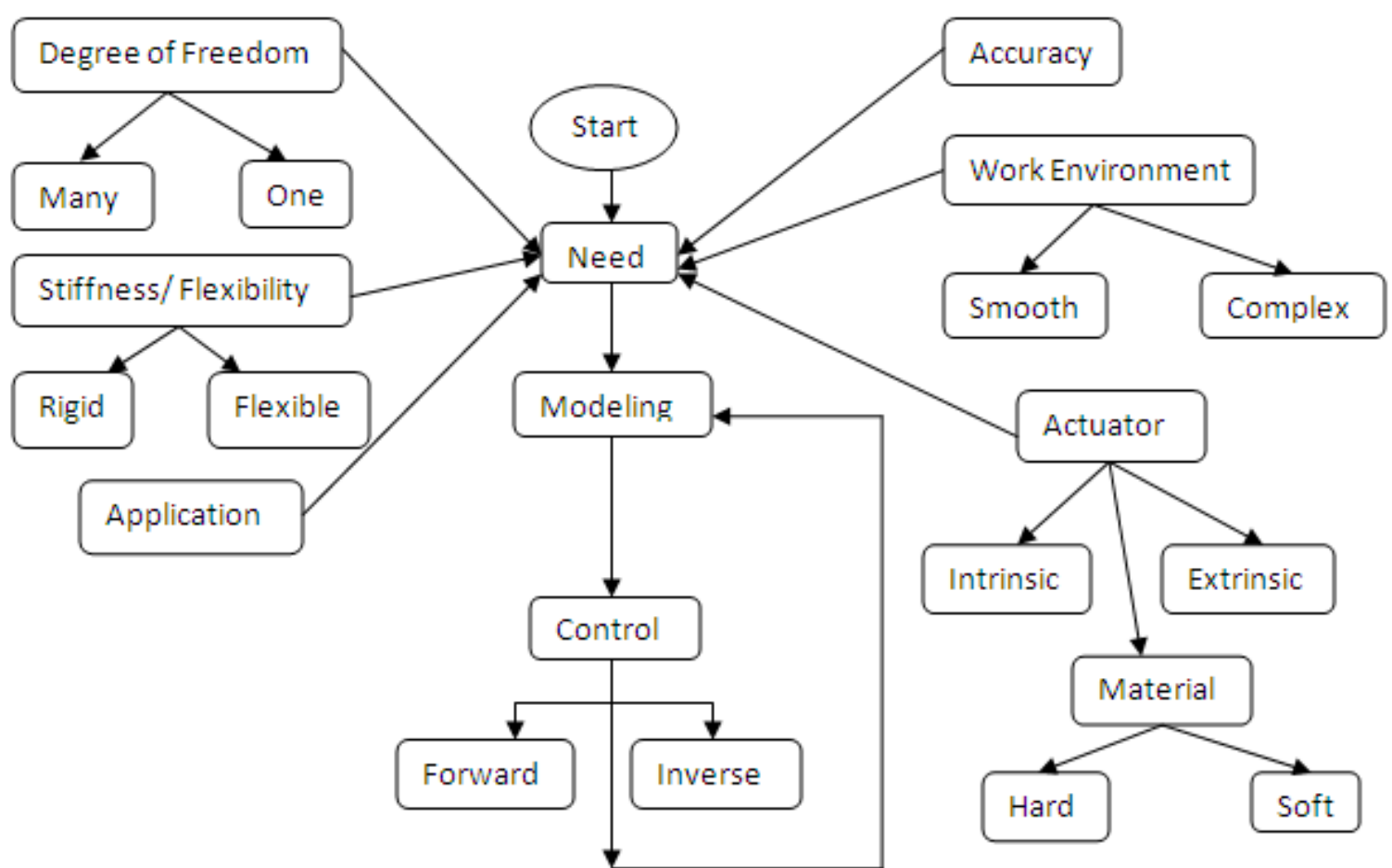

Figure 2. Flow chart for Design 


\section{Application (Bio-Inspired)}

\subsection{Medical}

Cannula is a tube that can be inserted into the body, often for the delivery or removal of fluid or for the gathering of data. Rucker et al.[1] by use of geometrically exact Kirchoff rod theory described the shape of a collection of concentric precurved tubes under general force and moment distributions and analyzes a collection of concentric precurved tubes under a general set of distributed and point wrenches made of Nitinol tubes and also presented a general coordinate-free energy formulation for modeling the shape of concentric tube continuum robots known as active cannulas. This formulation is able to account for pre curvatures and stiffness that vary along the length of component tubes, and to explicitly model torsion throughout the device. Ayvali et al.[2] presented a new and compact design of the discretely actuated cannula with antagonistic setup of shape memory alloy actuators. The design enables the placement of SMA actuators along the length of the cannula whereby each segment of the cannula is discretely actuated. CHEN et al.[3] designed micro-robotic tip for colonoscopy EDORA II by using Silicone rubber and developed control strategy for developed model. Simaan et al. [4] developed by using NiTi Tube of high dexterity, high accuracy slave for tele-operated minimally invasive surgery of the throat. The slave is a $34 \mathrm{DoF}$ unit capable of simultaneously manipulating up to three surgical tools. The design of the snake-like unit was presented. Butler et al.[14] presents a concentric tube manipulator mated to a robotically controlled flexible endoscope. The device adds three degrees of freedom to the standard neuroendoscope and roboticizes the entire package allowing the operator to conveniently manipulate the device. The flexible nature of endoscopes presents interesting problems in terms of robotic control. Bardou et al.[5] developed control for endoscope but the presence of important backlash induced by the cable transmission makes it challenging. They proposed an external sensor, the Aurora magnetic measurement system to provide feedback. Gayle et al.[6] presents a new algorithm for computing a collision free path for a deformable robot in a complex static environment. Sears et al.[7] proposed new approach to steerable needle design for use in minimally invasive surgery. The technology is based on sets of curved concentric tubes. By rotating and extending the tubes with respect to each other, the position and orientation of the needle tip, as well as the shape of the inserted length, can be controlled. A mechanics model is presented for computing the shape of the needle. Goksel et al.[8] presents three different models to simulate the deformations of a needle. The first two models use tetrahedral and beam elements, while the third model uses rigid bars connected through angular springs. Jung et al.[9] developed flexible robotic catheter systems for minimally invasive cardiac surgery. These systems commonly include a passive flexible catheter, which is biocompatible, and an active servo system which provides the actuation for the flexible catheter. Segreti et al.[10] presents a method for estimating drive cable length in an underactuated, hyper-redundant, snake-like manipulator. The continuum manipulator was designed for the surgical removal of osteolysis behind total hip arthroplasties. Camarillo et al.[11] presented a method for controlling a tendon-driven continuum manipulator by means of specifying the shape configuration. The basis for control is a linear beam configuration model that transforms beam configuration to tendon displacement by modeling internal loads of the compliant system. Dupont et al.[12]; Lock et al.[13] approached toward construction of robots based on a concentric combination of precurved elastic tubes. As the tubes are rotated and translated with respect to each other, their curvatures interact elastically, enabling control of the robot's tip configuration as well as the curvature along its length. Bai et al.[15] developed model of Concentric-tube continuum robots with a collection of super-elastic NiTiNol tubes. Mahvash et al.[16] introduces the first stiffness controller for continuum robots. The control law is based on an accurate approximation of a continuum robot's coupled kinematic and static force model.

\subsection{Industry}

Continuum robots offer the potential of robust grasps over a wide variety of object classes, due to their ability to adapt their shape to interact with the environment via non-local continuum contact conditions. Giri et al.[18] discusses the capabilities of continuum (continuous backbone) robot structures in the performance of under-actuated grasping. Gallardo et al.[17] presents an novel hyper-redundant manipulator. Such a manipulator is built with a variable number of tandem-assembled modules. Each module consists of a 3-dof parallel manipulator with asymmetric extremities in which moving platform possesses mixed motions with respect to the base platform. Zhao et al.[19] developed a new kind of bionic Continuum robot and presented a design scheme of Hook Joint type continuum robot. Jones et al.[20] presents both the mechanical design for a unique, variable-compliance 4 DOF robotic trunk and a set of forward and inverse kinematics which relate cable length to trunk length and curvature. The design is scalable from micro scale to tens of meters and covers a wide range of applications such as disaster relief, counter-terrorism, medical devices, and remote explorations. McMahan et al.[21] described the design and real-time implementation of a modular soft continuum manipulator, Air- Octor. The design is novel in combining both pneumatic and electric (tendon-driven) actuation within an internal/external hose structure. Neppalli et al.[22] developed a new design of continuum robot made of latex rubber and presents a unique rapid-prototyping system with which to operate the robot. Hannan et al.[24] presented a biological inspired hyper redundant manipulator. The developed elephant trunk robot is based on a highly maneuverable segmented backbone that contains four independently cable actuated sections. Hirose 
et al. [23] done biomechanical research on snakes and developmental research on snake-like robots and founded the technical difficulties in putting snake-like robots into practical.

\subsection{Rescue}

Folgheraiter et al.[25] presents the design concept for a bioinspired exoskeleton intended for applications in tele robotics and virtual reality also proposed a simplified kinematic model of the human arm using a notation coming from the robotics field. Hunt et al. [26] developed a robot for urban search and rescue missions. Tsukagoslii et al.[27] proposed both the design and driving mechanism of a flexible robot with multiple degrees of freedom to dive into debris, called "Active Hose," has multiple degrees of freedom by connecting units of two degrees freedom in series. Calisti et al.[28] described the design and development of a robot with six soft limbs, with the dual capability of pushing-based locomotion and grasping by wrapping around objects. The robot is inspired by the octopus and implements the key principles of locomotion in this animal. Siles et al.[29] introduces a novel mobile robot design concept aimed at Exploration of subterranean spaces. Pfeiffer et al.[30] described a feasibility study for the use of robotics in offshore oil and gas exploration and production facilities.

\subsection{Hazardous Place}

In order to increase the operating scope the robot has been made able to run round or over obstacles, to follow sewage branches and is aimed to work wirelessly unlike most other sewer inspection robots. Scholl et al.[31] presents a multi joint robot for sewer inspection tasks. Godage et al.[32] presents kinematics, dynamics, and experimental results for a novel quadruped robot using continuum limbs.

\subsection{Space}

Tendril robot is a long slender manipulator that can extend deep into crevasses and under thermal blankets to inspect areas largely inaccessible by conventional means. Inspired by the biology of snakes, tentacles, and climbing plants. Mehling et al.[33] developed Tendril, NASA's latest robot designed for minimally invasive inspection. Buckingham et al. [34] developed snake type commercial manipulator for OC Robotics.

\subsection{Underwater}

The octopus is a boneless animal and it's amazing dexterity is due to its muscular structure where longitudinal (axial), transverse (radial) and oblique muscles. Guglielmino et al. [35]designed 8 DOF robot inspired to the anatomy and morphology of an octopus arm. Zheng et al.[37] presents a kinematic and dynamic model for an underwater robotic manipulator inspired by Octopus vulgaris. Kang et al. [38] proposed a dynamic model for a multiple continuum arm robot inspired by live octopuses. The kinematics and dynamics for a single arm are analyzed and formulated including the longitudinal muscles, radial muscles, isovolumetric constraints, and interaction between suckers and an object. Zheng et al.[42] presents a kinematic and dynamic model for underwater continuum robots inspired by Octopus vulgaris. Mass, damping, stiffness, and external forces such as gravity, buoyancy, and hydrodynamic forces are considered in the dynamic model. Vavourakis et al.[40] addresses open-loop control strategies for continuum robotic manipulators inspired by the octopus arm, which are based on solving numerically a detailed elastodynamic model. Airacuda[36] is a remote controlled and pneumatically driven fish that consistently harnesses bionic principles. Khalil et al.[39] presents the dynamic modeling of a 3Dserial underwater eel-like robot using recursive algorithm based on the Newton-Euler equations. The proposed algorithm is easy to implement and to simulate whatever the number of degrees of freedom of the robot. Boyer et al.[41] presents the dynamic modeling of a continuous three-dimensional swimming eel-like robot. The modeling approach is based on the "geometrically exact beam theory" and on that of Newton-Euler.

\subsection{Military}

Lipkin et al.[43]described a series of gaits which are developed for a free crawling snake robot. Snake robots, a class of hyper-redundant mechanisms. Tatlicioglu et al. [44] derived a new dynamic model for continuum robot manipulators the dynamic model is developed based on the geometric model of extensible continuum robot manipulators with no torsional effects. Machiyamat et al. [45] discuss kinematics and dynamics of an HFM a Hyper-Flexible Manipulator is a kind of continuum robots with a simple mechanical structure like a cable, rope and string, which are useful tools utilized everywhere in various forms. They also derive a spatial, nonlinear and continuum dynamics model with an under actuated mechanism using special kinematics based on curve geometry and theory of robot manipulation.

\section{Conclusion}

The continued advancement of soft robotics depends on the development of novel soft sensors and actuators, soft robot designs with mobility and strength, models that enable design optimisation and control and fabrication techniques that build active soft structures and interconnections. Active materials currently available for use in soft robotic manipulators have shortcomings that make their commercial use impractical. New active materials are needed that provide the strain, stress and speed for this challenging application. Material science and engineering is required to 
produce bulk quantities of high quality active material and reliably fabricate high-performance actuators and sensors. Biological continuum manipulators mostly rely on hard/discrete elements in their structure and/or operation to reduce complexity in interaction with their environment, and similarly, a judicious mixture of continuous/soft and discrete/hard elements would significantly improve the performance of these robots in most applications. To enable rapid virtual prototyping of soft robots, accurate physical models are needed. Development of models that accurately simulate the operation of these robots on the basis of the actuation inputs is a challenging multiphysics problem that can involve simultaneous analysis of solid and fluid mechanics, kinematics, electromechanics, thermodynamics and chemical kinetics of the processes involved. New approaches in path planning that are computationally efficient for deformable bodies with infinite DOF are required to make real-time path-planning viable. Integrating motion planning with control and sensing is also an open problem. Grasping objects by using whole arm manipulation requires the grasp to be stable so that the arm does not undergo sudden changes in shape and drop the manipulated object. Robust path-planning and control algorithms must ensure the stability of all intermediate configurations of the manipulator along the prescribed path. Extensive literature exists for grasp synthesis and stability analysis of rigid-link robots that contact objects only at finite points Stable grasp synthesis for highly compliant and continuously deformable soft manipulators, however, will require solutions to many untouched and challenging design, control and planning problems.

\section{REFERENCES}

[1] D. Caleb Rucker, Bryan A. Jones and Robert J. Webster III (2010) A Geometrically Exact Model for Externally Loaded Concentric-Tube Continuum Robots, IEEE Transactions on Robotics, Vol. 26, No. 5

[2] Elif Ayvali and Jaydev P. Desai (2012) Towards A Discretely Actuated Steerable Cannula, IEEE International Conference on Robotics and Automation

[3] Gang CHEN (2005) Design, modeling and control of a micro-robotic tip for Colonoscopy, Numéro d'ordre : -ISAL-00107

[4] Nabil Simaan, Russell Taylor and Paul Flint (2004) A Dexterous System for Laryngeal Surgery, Proceeding of the IEEE International conference on Robotic \& Automation

[5] B'ereng'ere Bardou, Philippe Zanne, Florent Nageotte and Michel de Mathelin (2010) Control of a Multiple Sections Flexible Endoscopic System, The IEEE/RSJ International Conference on Intelligent Robots and Systems

[6] Russell Gayle, Paul Segars, Ming C. Lin, and Dinesh
Manocha (2005) Path Planning for Deformable Robots in Complex Environments, gamma.cs.unc.edu/FlexiPlan/M edia/robotics_final.pdff

[7] Patrick Sears and Pierre Dupont; A Steerable Needle Technology Using Curved Concentric Tubes (2006) Proceedings of the IEEE/RSJ International Conference on Intelligent Robots and Systems

[8] Orcun Goksel, Ehsan Dehghan, Septimiu E. Salcudean (2009) Modeling and simulation of flexible needles, Medical Engineering \& Physics 31 1069-1078

[9] Jinwoo Jung, Ryan S. Penning, Nicola J. Ferrier and Michael R. Zinn (2011) A Modeling Approach for Continuum Robotic Manipulators: Effects of Nonlinear Internal Device Friction, IEEE/RSJ International Conference on Intelligent Robots and Systems

[10] Sean M. Segreti, Michael D. M. Kutzer, Ryan J. Murphy, and Mehran Armand (2012) Cable Length Estimation for a Compliant Surgical Manipulator, IEEE International Conference on Robotics and Automation

[11] David B. Camarillo, Christopher R. Carlson and J. Kenneth Salisbury (2009) Configuration Tracking for Continuum Manipulators With Coupled Tendon Drive; IEEE Transactions on Robotics, Vol. 25, No. 4

[12] Pierre E. Dupont, Jesse Lock, Brandon Itkowitz and Evan Butler (2010) Design and Control of Concentric-Tube Robots; IEEE Transactions on Robotics, Vol. 26, No. 2

[13] Jesse Lock, Genevieve Laing, Mohsen Mahvash and Pierre E. Dupont (2010) Quasistatic Modeling of Concentric Tube Robots with External Loads, The IEEE/RSJ International Conference on Intelligent Robots and Systems

[14] Evan J. Butler, Robert Hammond-Oakley, Szymon Chawarski, Andrew H. Gosline, Patrick Codd, Tomer Anor, Joseph R. Madsen, Pierre E. Dupont and Jesse Lock (2012) Robotic Neuro Endoscope with Concentric Tube Augmentaion, IEEE/RSJ International Conference on Intelligent Robots and Systems

[15] Shaoping Bai and Chuhao Xing (2012) Shape Modeling of a Concentric-tube Continuum Robot, Proceedings of the IEEE International Conference on Robotics and Biomimetics

[16] Mohsen Mahvash and Pierre E. Dupont, (2011) Stiffness Control of Surgical Continuum Manipulators, IEEE Transactions on Robotics, Vol. 27, No. 2

[17] Jaime Gallardo, Horacio Orozco, Jose M. Rico, EmilioJ.Gonza lez-Galva (2009) A new spatial hyper-redundant manipulator, Robotics and Computer Integrated Manufacturing 25 703-708

[18] N. Giri and I.Walker (2011) Continuum robots and under actuated grasping, Mech. Sci., 2, 51-58

[19] Qiang Zhao and Fang Gao (2010) Design and Analysis of a Kind of Biomimetic Continuum Robot; Proceedings of the 2010 IEEE International Conference on Robotics and Biomimetics

[20] Bryan A. Jones, William McMahan , and Ian Walker (2004) Design and Analysis of A Novel Pneumatic Manipulator, 
www.clemson.edu/ces/crb/octor/publications/ifac_2004_ submission.pdf

[21] William McMahan, Bryan A. Jones, and Ian D. Walker (2005) Design and Implementation of a Multi-Section Continuum Robot: Air-Octor, IROS

[22] Srinivas Neppalli and Bryan A. Jones (2007) Design, Construction, and Analysis of a Continuum Robot, Proceedings of the IEEE/RSJ International Conference on Intelligent Robots and Systems

[23] Shigeo Hirose and Hiroya Yamada (2009) Snake-Like Robots; IEEE Robotics \& Automation Magazine

[24] M. W. Hannan and I .D. Walker (2001) The 'Elephant Trunk' Manipulator , Design and Implementation , www.clemson.edu/ces/crb/students/mhannan/pdf.../hann an_aim.pdf, 2001

[25] Michele Folgheraiter, Bertold Bongardt, Jan Albiez, Frank Kirchner (2008) A Bio-Inspired Haptic Interface for Tele-Robotics Applications; Proceedings of the IEEE International Conference on Robotics and Biomimetics

[26] Alexander J. Hunt, Richard J. Bachmann, Robin R. Murphy and Roger D. Quinn (2011) A Rapidly Reconfigurable Robot for Assistance in Urban Search and Rescue, IEEE/RSJ International Conference on Intelligent Robots and Systems

[27] Hideyuki Tsukagoslii, Ato Kitagawa, Mitsuru Segawa (2001) Active Hose: an Artificial Elephant's Nose with Maneuverability for Rescue Operation; Proceedings of the IEEE, International Conference on Robotics \& Automation

[28] Marcello Calisti, Andrea Arienti, Federico Renda, Guy Levy, Binyamin Hochner, Barbara Mazzolai, Paolo Dario, and Cecilia Laschi (2012) Design and development of a soft robot with crawling and grasping capabilities, IEEE International Conference on Robotics and Automation

[29] Ivan Siles, Ian D. Walker (2009) Design, Construction, and Testing of A New Class of Mobile Robots for Cave Exploration;

www.ces.clemson.edu/ ianw/Siles_ICM2009.pdff

[30] K. Pfeiffer, M. Bengel, A. Bubeck (2011) Offshore Robotics - Survey, Implementation, Outlook, IEEE/RSJ International Conference on Intelligent Robots and Systems

[31] K.-U. Scholl, V. Kepplin, K. Berns, R. Dillmann (2000) Controlling a Multijoint Robot for Autonomous Sewer Inspection; Proceedings of the IEEE Intemational Conference on Robotics \& Automation

[32] Isuru S. Godage, Thrishantha Nanayakkara, and Darwin G. Caldwell (2012) Locomotion with Continuum Limbs, IEEE/RSJ International Conference on Intelligent Robots and Systems

[33] Joshua S. Mehling, Myron A. Diftler, Mars Chu and Michael Valvo (2006) A Minimally Invasive Tendril Robot for In-Space Inspection; The First IEEE/RAS-EMBS International Conference on Biomedical Robotics and Biomechatronics
[34] Rob Buckingham and Andrew Graham (2000) Reaching the unreachable - snake-arm robots,;OCRobotics Ltd, UK

[35] Emanuele Guglielmino, Nikos Tsagarakis, Darwin G. Caldwell (2010) An Octopus Anatomy-inspired Robotic Arm; The IEEE/RSJ International Conference on Intelligent Robots and Systems

[36] AIRACUDA, (2006) www.festo.com/rep/en_corp/assets /pdf/Airacuda_en.pdff

[37] Tianjiang Zheng, David T. Branson III, Rongjie Kang, Matteo Cianchetti, Emanuele Guglielmino, Maurizio Follador, Gustavo A. Medrano-Cerda, Isuru S. Godage and Darwin G. Caldwell (2012) Dynamic Continuum arm Model for Use with Underwater Robotic Manipulators Inspired by Octopus vulgaris, IEEE International Conference on Robotics and Automation

[38] Rongjie Kang, David T. Branson, Emanuele Guglielmino, Darwin G. Caldwell (2012) Dynamic modeling and control of an octopus inspired multiple continuum arm robot; Computers and Mathematics with Applications 64, 1004-1016

[39] Wisama Khalil, Guillaume Gallot, Ouarda Ibrahim and Frédéric Boyer (2005) Dynamic Modeling of a 3-D Serial Eel-Like Robot; Proceedings of the IEEE International Conference on Robotics and Automation

[40] Vasileios Vavourakis, Dimitris Bampasakis, Asimina Kazakidi, Nikolaos Pateromichelakis, John A. Ekaterinaris and Dimitris P. Tsakiris (2012) Generation of Primitive Behaviors for Non-Linear Hyperelastic Octopus-inspired Robotic Arm; The Fourth IEEE RAS/EMBS International Conference on Biomedical Robotics and Biomechatronics

[41] Frédéric Boyer, Mathieu Porez, and Wisama Khalil (2006) Macro-Continuous Computed Torque Algorithm for a Three-Dimensional Eel-Like Robot, IEEE Transactions on Robotics, Vol. 22, No. 4

[42] Tianjiang Zheng, David T. Branson, Emanuele Guglielmino, Rongjie Kang, Gustavo A. Medrano Cerda, Matteo Cianchetti, Maurizio Follador, Isuru S. Godage and Darwin G. Caldwell (2013) Model Validation of an Octopus Inspired Continuum Robotic Arm for Use in Underwater Environments; Journal of Mechanisms and Robotics, Vol. 5 / 021004-1

[43] Kevin Lipkin, Isaac Brown, Aaron Peck, Howie Choset, Justine Rembisz, Philip Gianfortoni, Allison Naaktgeboren (2007) Differentiable and Piecewise Differentiable Gaits for Snake Robots; Proceedings of the IEEE/RSJ International Conference on Intelligent Robots and Systems

[44] Enver Tatlicioglu, Ian D. Walker, and Darren M. Dawson (2007) Dynamic Modelling for Planar Extensible Continuum Robot Manipulators; IEEE International Conference on Robotics and Automation

[45] Hiromi Machiyamat and Takahiro Suzukit (2003) Kinematics and Dynamics of a Cable-like Hyper-flexible Manipulator ; Proceeding of ICRA. 